Mappemonde

Revue trimestrielle sur l'image géographique et les

formes du territoire

$131 \mid 2021$

Varia

\title{
Géovisualisation des flux de transport aérien entre 1999 et 2020 : de la donnée brute à la création d'un récit interactif
}

Tony Hauck

\section{(2) OpenEdition}

1 Journals

Édition électronique

URL : https://journals.openedition.org/mappemonde/6300

DOI : $10.4000 /$ mappemonde. 6300

ISSN : 1769-7298

Éditeur

UMR ESPACE

Référence électronique

Tony Hauck, «Géovisualisation des flux de transport aérien entre 1999 et 2020 : de la donnée brute à la création d'un récit interactif », Mappemonde [En ligne], 131 | 2021, mis en ligne le 08 juillet 2021, consulté le 14 juillet 2021. URL : http://journals.openedition.org/mappemonde/6300 ; DOI : https:// doi.org/10.4000/mappemonde.6300

Ce document a été généré automatiquement le 14 juillet 2021.

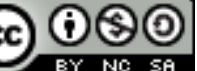

La revue Mappemonde est mise à disposition selon les termes de la Licence Creative Commons Attribution - Pas d'Utilisation Commerciale - Partage dans les Mêmes Conditions 4.0 International. 


\title{
Géovisualisation des flux de transport aérien entre 1999 et 2020 : de la donnée brute à la création d'un récit interactif
}

\author{
Tony Hauck
}

\section{Introduction}

1 Ce texte présente la datavisualisation narrative des dynamiques spatio-temporelles du transport aérien entre 1999 et 2020 en France métropolitaine. Cette datavisualisation a été soumise dans le cadre du Mapathon, un concours de géovisualisation organisé pour le colloque Tous (im)mobiles, tous cartographes? (http://cartomob.sciencesconf.org/).

2 À l'heure actuelle, l'importance du transport aérien est une puissante manifestation du "système-monde », concept imaginé par Olivier Dollfus (Dollfus, 1984) au milieu des années 80. À cet égard, les dynamiques des flux aériens en France ne seraient qu'une des nombreuses ramifications de ce système. C'est tout l'objectif de ce travail : tenter de rendre compte des mouvements aériens à l'œuvre sur le territoire français par la datavisualisation.

\section{Données}

3 Plusieurs jeux de données sur l'évolution spatio-temporelle du transport aérien (passagers, fret et courrier) observée en France métropolitaine ont été fournis. Ces données ont été collectées par les organisateurs auprès de la DGAC ${ }^{1}$ et d'Eurostat.

Elles portaient à la fois sur les villes aéroportuaires et sur les flux entre ces différentes localisations. Seules les métropoles présentant un aéroport en activité depuis 2010 sont concernées. Les villes telles que Paris et Lyon, qui présentent plusieurs aéroports, ont 
vu leurs statistiques fusionner. À noter que ces chiffres décrivaient non pas des flux orientés d'une ville vers une autre, mais bien le cumul du trafic dans les deux sens.

\section{Méthodes et concepts monopolisés}

\section{Le scrollytelling : une nouvelle manière de consommer de l'information}

5 Les NTIC (Nouvelles Technologie d'Information et de Communication) ont vu apparaître avec elles de nouvelles pratiques de consommation de l'information. Parmi celles-ci nous trouvons le scrollytelling. Mot valise provenant de la contraction des mots scrolling et storytelling, ce concept représente une manière plus dynamique de raconter des histoires et de mêler contenus multimédias et narration. Ce format a rencontré un vif succès, notamment dans le secteur du journalisme (New York Times, Washington Post, Les Échos, The Guardian...). Ces grands quotidiens ont réussi leur pari d'impacter le lecteur avec ce nouveau genre de datavisualisation mêlant narration maitrisée, contenus graphiques qualitatifs et déroulé simple et cohérent. C'est notamment une datavisualisation du New York Times sur la diffusion du coronavirus, où la propagation à partir de la Chine est traduite par les trajectoires animées de particules, qui a inspiré ce travail («How the Virus Got Out », NYTimes, 22 mars $2020^{2}$.

Différentes bibliothèques JavaScript permettent d'importer ce genre de narration dans ses propres productions numériques ${ }^{3}$. La présente réalisation se base sur scrollama.js, développée par Russell Goldenberg. Elle a été choisie pour sa simplicité d'utilisation.

Enfin, sur le fond, nous nous sommes basés sur l'Homme de Vitruve, le célèbre dessin annoté de De Vinci, pour poser les bases de notre narration et garder l'attention du lecteur.

\section{Les apports du game design dans le scrollytelling : la mécanique du tutoriel}

8 Le jeu vidéo, d'une manière générale, a énormément à apporter à la datavisualisation. En effet, ces deux médiums poursuivent un intérêt commun : immerger le joueur/lecteur dans un univers et retenir son attention. C'est dans cette optique que le concept du tutoriel $^{4}$ a été monopolisé pour ce travail. Le tutoriel est une phase du jeu ayant pour but d'expliquer au joueur toutes les fonctionnalités essentielles au bon déroulement de sa partie. Ce travail tente de transposer dans la datavisualisation trois règles de base propres à tout bon tutoriel :

\begin{tabular}{|l|l|}
\hline TUTORIEL & DATAVISUALISATION \\
\hline $\begin{array}{l}\text { L'enseignement des fonctionnalités au } \\
\text { moment où le joueur en a besoin. }\end{array}$ & $\begin{array}{l}\text { C'est le niveau de scroll qui définira l'apparition } \\
\text { d'une nouvelle fonctionnalité d'interaction } \\
\text { (déblocage du clic sur les cercles, information au } \\
\text { survol de la carte...) }\end{array}$ \\
\hline
\end{tabular}


La découverte de nouvelles mécaniques de jeu (jusqu'alors masquées) au fur et à mesure que la narration progresse est un facteur de motivation.

Un déroulé ludique où chaque action entraîne récompenses et feedbacks (retours visuels).
Les filtres, les graphiques ou tout autre élément d'interaction seront uniquement dévoilés lorsqu'un niveau précis du scroll sera atteint.

Exemple: les clics sur les cercles ont pour effet de filtrer les particules visibles sur une carte, de faire apparaître un graphique sur le volet droit...

Tout cela aura pour bénéfice d'amener le lecteur plus rapidement au cœur de la datavisualisation et du propos développé.

\section{La visualisation}

10 La difficulté principale de ce type d'exercice réside dans la visualisation combinée des dimensions temporelle et spatiale d'une entité cartographique difficile à appréhender et à représenter : le flux. Le flux « est la mesure d'une quantité transférée depuis un lieu d'Origine (i) vers un lieu (j) de Destination (OD), pendant une période $(\mathrm{t})$ » (Bahoken et al., 2016, p. 66).

\section{Du flux au mouvement animé}

11 L'approche classique en cartographie consiste à représenter les flux selon une largeur, une longueur et une surface, la largeur du flux variant proportionnellement à sa valeur. Ici, les flux n'étant pas unidirectionnels, mais bien bidirectionnels (dans la mesure où la donnée fournie concerne des liaisons), nous avons souhaité explorer une nouvelle voie dans leur visualisation, en travaillant le flux de deux manières :

- Faire varier l'importance du flux selon un degré d'opacité de la couleur en nous basant sur la perception visuelle du lecteur et sa capacité à hiérarchiser l'importance des liaisons selon leur intensité colorimétrique.

- Éclater les flux en un ensemble de particules permettant de simuler le trafic aérien sur le territoire.

Pour répondre à ces deux objectifs, trois bibliothèques JavaScript ont été utilisées : Mapbox pour le volet cartographique, three.js pour la modélisation du trafic aérien et d3.js pour la représentation des villes sous la forme de cercles proportionnels.

\section{Dimension spatiale du flux}

Mapbox GL ainsi que Mapbox Studio nous ont servi à produire l'ensemble de l'architecture cartographique. En effet, nous avons produit un fond de carte interactif propre au projet, en travaillant sur Mabpox studio (figure 1), une interface permettant de produire ses propres basemaps (fonds de carte). 
Figure 1. Création du fond de carte sous Mapbox Studio

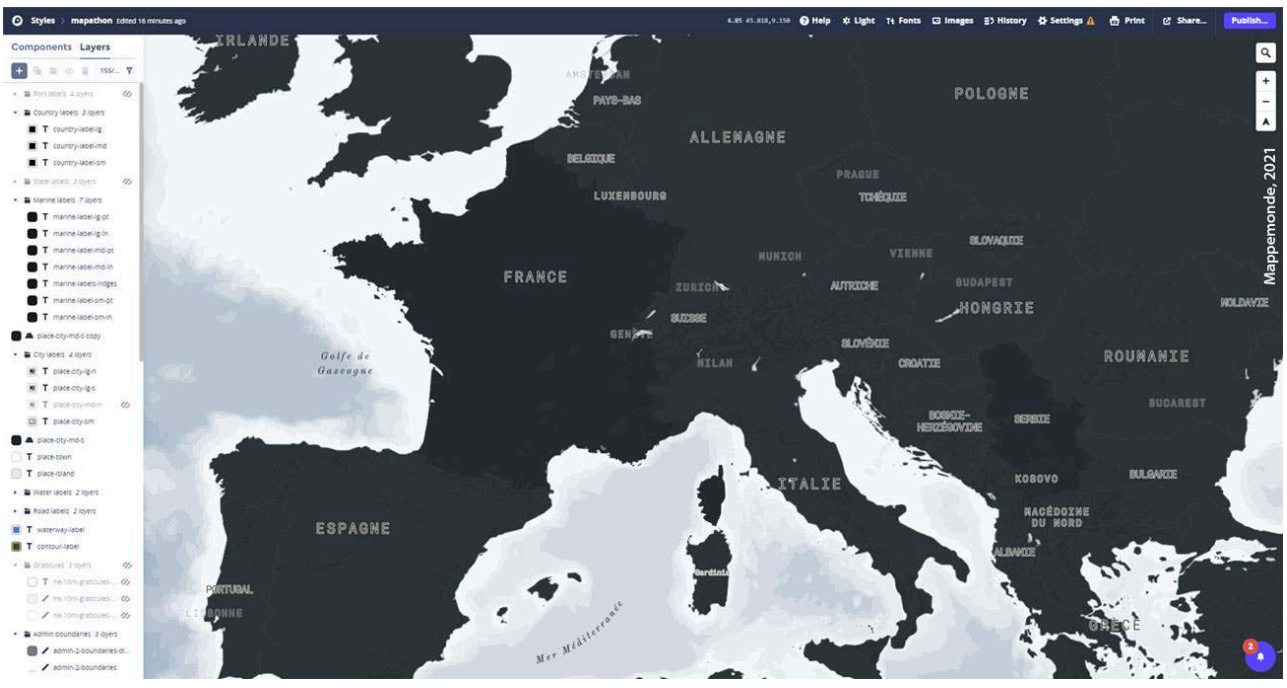

15 Cette librairie a été couplée à D3.js, une bibliothèque de datavisualisation, qui se charge de calculer et d'afficher les diamètres des cercles selon la méthode des racines carrées (square root ou d3.scaleSqrt) qui nous donnait une perception plus correcte des valeurs représentées.

Les particules ont été générées grâce à three.js, une bibliothèque JavaScript pour créer des scènes 3D dans un navigateur web. La quantité de particules affichées pour chacun des flux est proportionnelle à sa valeur. De la même manière, dans l'optique d'améliorer la lisibilité de la dataviz, un filtre de visualisation permet de sélectionner uniquement les liaisons concernées par une ville de départ (figure 2).

Figure 2. Les flux peuvent être filtrés par ville

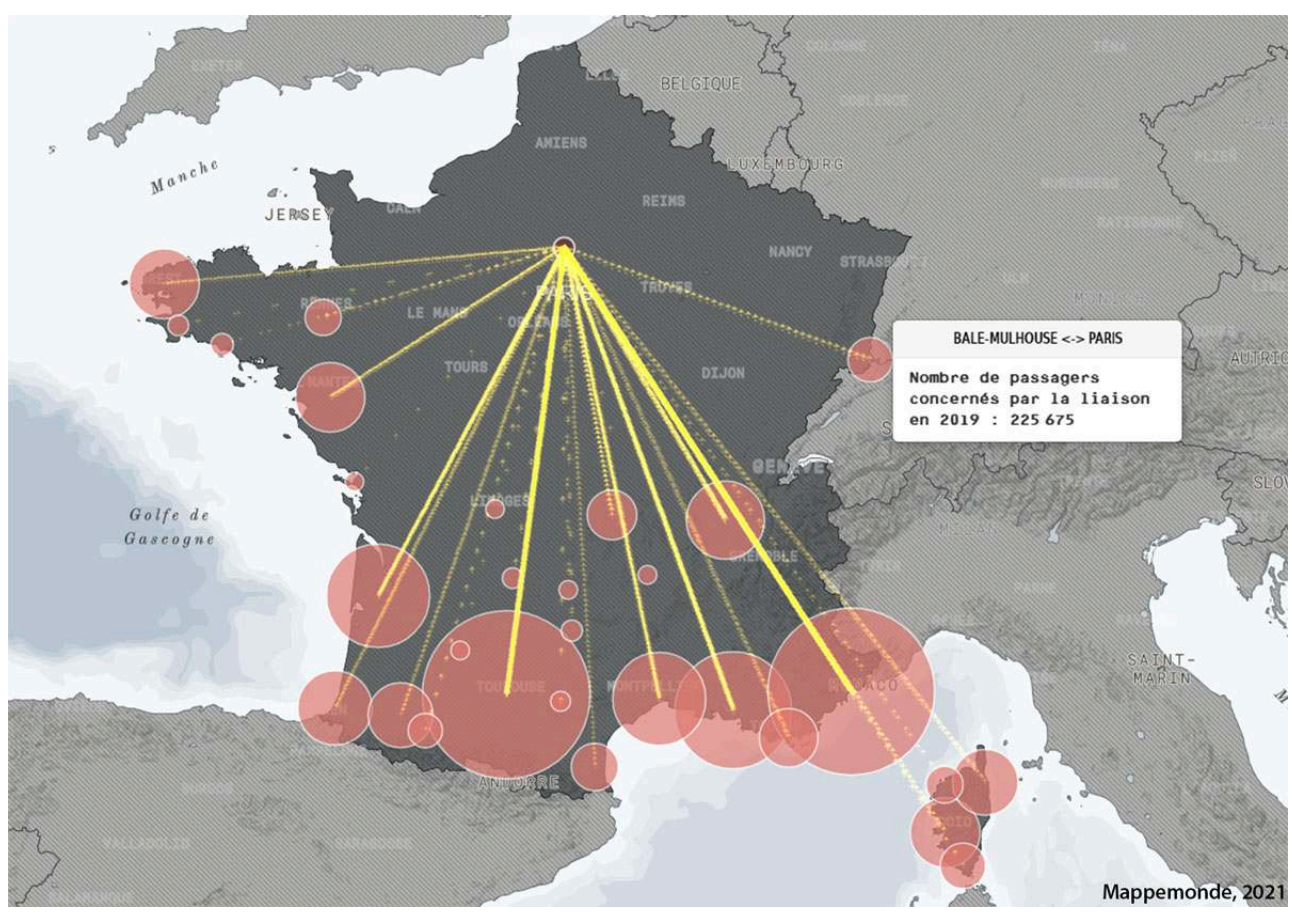




\section{Dimension temporelle}

17 La dimension temporelle peut être retrouvée dans les différentes ramifications de la datavisualisation, comme sur la carte, où nous pourrons voir l'évolution de chaque flux par le biais d'un sélecteur d'années.

18 Sur l'exemple ci-dessous (figure 3), l'intensité colorimétrique et la représentation en particules nous permettent d'apprécier l'augmentation des flux liés au transport de marchandises entre 1999 et 2019.

Figure 3. Visualisation des flux de marchandises en 1999 (à gauche) et en 2019 (à droite)

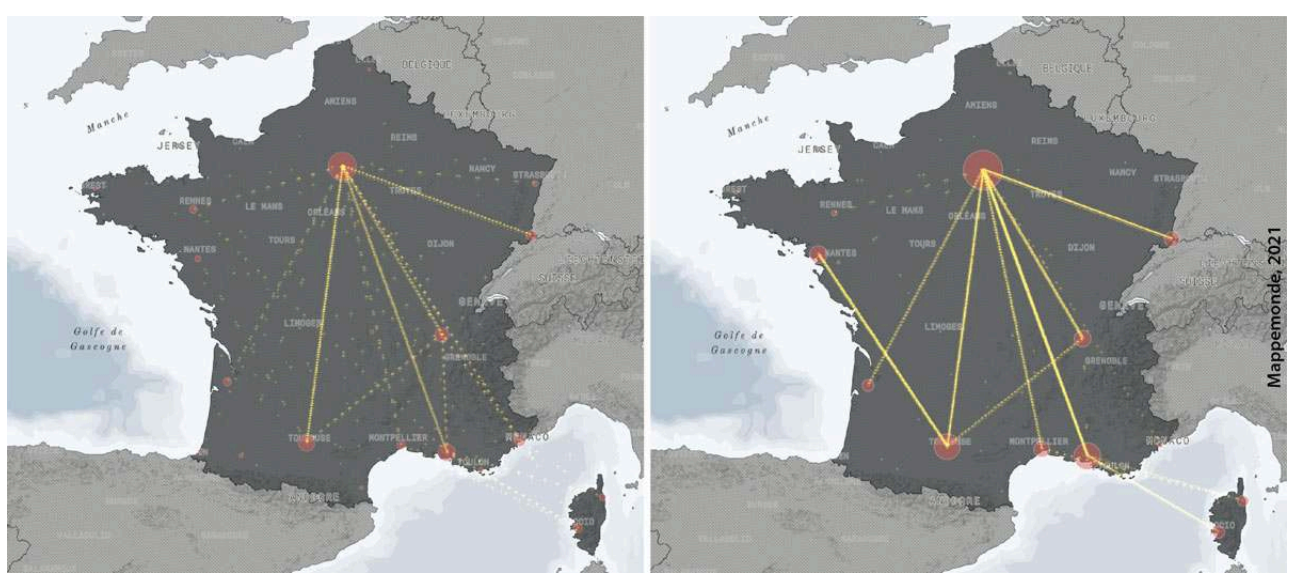

Enfin, la génération d'un streamgraph (figure 4) nous donne plusieurs informations concrètes, à la fois sur son attractivité dans le temps, et sur l'évolution du nombre de passagers ou de marchandises pour la ville concernée et ses liaisons entre 1999 et 2019. Ce streamgraph est produit avec highchart.js, une bibliothèque JavaScript relativement simple à utiliser pour la production de graphiques interactifs. 
Figure 4. Le streamgraph nous donne une information temporelle et spatiale sur la ville sélectionnée

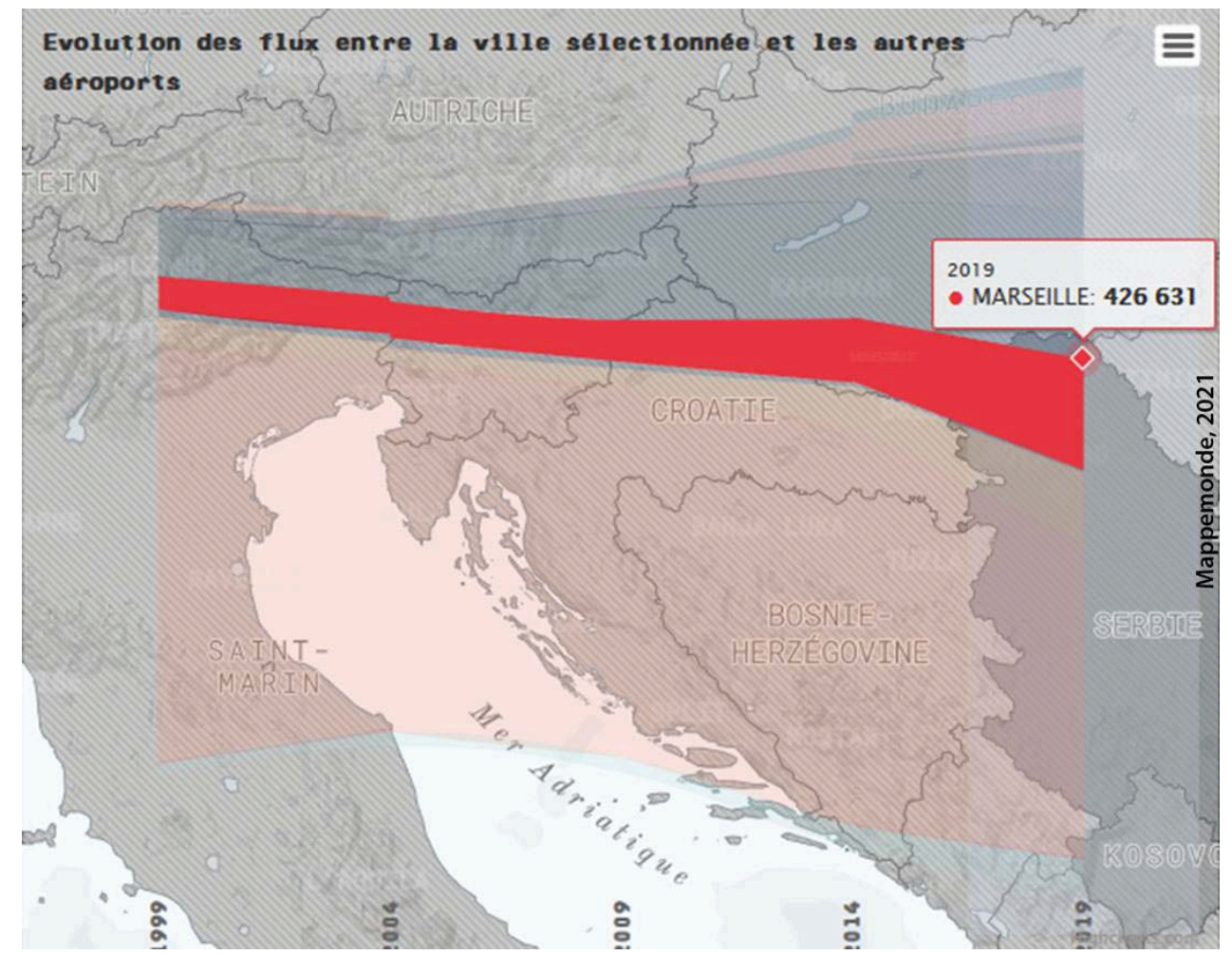

\section{La cohérence du fond avec la forme}

Cette datavisualisation cherche à concilier la forme avec le propos de base : le trafic aérien. Plusieurs éléments graphiques permettent de faire le pont entre ces deux volets. Tout d'abord, le fond cartographique dessiné emprunte des détails visuels présents dans les anciennes cartes présentant les destinations desservies par les compagnies aériennes, comme, par exemple, une carte de 1986 de la Continental Airlines «The World According to Continental » (airlinetimetables.blogspot.com/2018/12/ continental-airlines-september-1986.html).

On pensera ainsi à l'utilisation d'un fond carroyé, à l'utilisation de couleur pâle ou foncée (permettant de faire ressortir les cercles proportionnels rosés et les particules jaunes), à un design très épuré pour le fond de carte, et à l'utilisation de polices de caractère caractéristiques de cette époque (Roboto Mono Light, combiné à un halo blanc autour des caractères pour les libellés de pays ; Old Standard TT Italic, pour les libellés associés au milieu marin). Le popup est également une référence à un billet d'avion, tant sur sa forme que sur la typographie adoptée (F25 Bank Printer).

Enfin, les particules d'avions survolant le territoire vont dans le sens d'une schématisation du trafic aérien français. Malheureusement, quelques bugs d'affichage et de rafraîchissement de ces particules peuvent venir ternir l'expérience narrative.

\section{En conclusion}

Les nouveaux outils numériques à notre disposition nous donnent la possibilité de visualiser les flux d'une manière différente. Ces innovations doivent permettre à 
l'utilisateur d'améliorer la perception visuelle des flux tout en gardant une logique sémiologique cohérente.

\section{BIBLIOGRAPHIE}

BAHOKEN F., GRASLAND C., ZANIN C. (2016). «D'une cartographie de flux à une cartographie du mouvement. Aspects sémiologiques ». Cartes et Géomatique, p. 65-74.

DOLLfUS O. (1984). «Le Système Monde : proposition pour une étude de géographie ». Géopoint 84, p. 231-239.

GENVO S. (2006). Le game design de jeux vidéo : une approche communicationnelle et interculturelle. Thèse en Sciences de l'information et de la communication. Université Paul Verlaine - Metz.

\section{NOTES}

1. Direction Générale de l'Aviation Civile.

2. www.nytimes.com/interactive/2020/03/22/world/coronavirus-spread.html

3. Présentation de plusieurs librairies de scrollytelling: https://pudding.cool/process/how-toimplement-scrollytelling/

4. Système de règles, une structure imposée au joueur pour l'amener vers une situation ludique (Genvo, 2006). 